\title{
The Effects of Multi-Modality on the Use of Smart Phones
}

\author{
Gaeun Lee ${ }^{1}$, Seongmin Kim¹, Jaeho Choe ${ }^{2}$, Eui Seung Jung ${ }^{1}$ \\ ${ }^{1}$ Department of Industrial Management Engineering, Korea University, Seoul, 136-713 \\ ${ }^{2}$ Department of Industrial \& Management Engineering, Daejin University, Pocheon, 487-711
}

\section{Corresponding Author}

Eui Seung Jung

Department of Industrial Management Engineering, Korea University, Seoul,

\section{6-713}

Mobile : $+82-10-8746-3391$

Email : ejung@korea.ac.kr

Received : April 29, 2014

Revised : May 14, 2014

Accepted: May 19, 2014
Objective: The objective of this study was to examine multi-modal interaction effects of input-mode switching on the use of smart phones.

Background: Multi-modal is considered as an efficient alternative for input and output of information in mobile environments. However, there are various limitations in current mobile UI (User Interface) system that overlooks the transition between different modes or the usability of a combination of multi modal uses.

Method: A pre-survey determined five representative tasks from smart phone tasks by their functions. The first experiment involved the use of a uni-mode for five single tasks; the second experiment involved the use of a multi-mode for three dual tasks. The dependent variables were user preference and task completion time. The independent variable in the first experiment was the type of modes (i.e., Touch, Pen, or Voice) while the variable in the second experiment was the type of tasks (i.e., internet searching, subway map, memo, gallery, and application store).

Results: In the first experiment, there was no difference between the uses of pen and touch devices. However, a specific mode type was preferred depending on the functional characteristics of the tasks. In the second experiment, analysis of results showed that user preference depended on the order and combination of modes. Even with the transition of modes, users preferred the use of multi-modes including voice.

Conclusion: The order of combination of modes may affect the usability of multimodes. Therefore, when designing a multi-modal system, the fact that there are frequent transitions between various mobile contents in different modes should be properly considered.

Application: It may be utilized as a user-centered design guideline for mobile multi modal UI system.

Keywords: Mobile, Multi-modal interaction, Mode-switching, Multi-modality, Voice

\section{Introduction}

of Korea. All right reserved.

(c) This is an open-access article distributed under the terms of the Creative Commons Attribution Non-Commercial License (http:// creativecommons.org/licenses/by-nc/3.0/), which permits unrestricted non-commercial use, distribution, and reproduction in any medium, provided the original work is properly cited.
Multimodal systems process two or more combined user input-modes such as speech, pen, touch, manual gestures, gaze, and head and body movements, etc. (Oviatt et al., 2000). There are many advantages for a multimodal use. First, multimodal interface can accommodate a wide range of users. Multimodal interfaces are relatively easy to learn and use, and are preferred by users for many applications. Multimodal 
interaction gives the user the freedom to interact with a system via more than a single human sense. Second, multimodal interfaces permit flexible use of input modes in a variety of user context. Third, when an error occurs, multimodal interface should be designed to include complementary input modes, so the alternative input modes can provide duplicate functionality such that users canaccomplish their goals using either mode. It means multimodal interface can be handled more efficiently rather than uni-modal interface (Oviatt and Olsen, 1994; Oviatt et al., 2000).

The mobile environment can be limited by various environmental constraints such as size and space, etc. For these kinds of environmental issues, using single sense input does not satisfy user's needs. Also, multi-tasking is required for the various contents efficiency in mobile environment. In terms of information processing, multi-modality can be interpreted as sequential processing in time or one at a time (Trouvain and Christopher, 2007; Wickens, 2002; Yang and Jung, 2012). Multi-modal in mobile can be classified into touch interface with the keyboard input method, voice, pen recognizing handwritten. Touch and pen modes are classified in the same category as motion-based, however, they have different characteristics from each other (Park et al., 2006).

When switching contents in smart phones, usability can vary by the order and combination of modes. Therefore, combinations of modes must be empirically investigated from the user's point of view from experiments. This study tried to verify smart phone usability through a combination of function and modes.

\section{Method}

\subsection{Research procedure}

The objective of this study was to examine multi-modal interaction effects of input-mode switching on the use of smart phones. First, a pre-survey determined five representative tasks from smart phone tasks by their functions. Second, the uni-mode experiment verified the effect of uni-mode according to the function characteristic of smart phone. And the high preference mode is derived from the first experiment. Lastly, the effect of alternative multi-modes is verified when task was sequentially processed.

\subsection{Classification of the tasks in smart phone}

On the basis of existing functions that were extracted from 2013 smart phones, 25 functions were selected and analyzed by factors analysis. From the eigenvalues with a value greater than 1.0 often interpreted as being meaningful, the five factors were derived from the analysis, as shown in Table 1 . The analysis was used to determine the number of tasks in smart phones that were extracted from the data. A task that was representative was selected for each factor. Five representative tasks from the classified groups of smart phone tasks are shown in Table 2.

Table 1. Results of factor analysis for smart phone tasks

\begin{tabular}{l|l|c}
\hline Factor & \multicolumn{1}{|c|}{ Tasks } & Loadings \\
\hline \multirow{2}{*}{ Factor 1 } & Internet searching & 0.739 \\
\cline { 2 - 3 } & Text messages (SMS, etc.) & 0.662 \\
\hline \multirow{2}{*}{ Factor 2 } & Subway map & 0.767 \\
\hline \multirow{2}{*}{ Factor 3 } & Memo & 0.846 \\
\cline { 2 - 3 } & E-mail & 0.743 \\
\cline { 2 - 3 } & Diet, Fitness & 0.714 \\
\hline
\end{tabular}


Table 1. Results of factor analysis for smart phone tasks (Continued)

\begin{tabular}{l|l|c}
\hline Factor & Tasks & Loadings \\
\hline \multirow{2}{*}{ Factor 4 } & Gallery (photo, video) & 0.893 \\
\cline { 2 - 3 } & Education and learning & 0.803 \\
\hline Factor 5 & Application store & 0.921 \\
\hline
\end{tabular}

Table 2. Tasks selected for experiment

\begin{tabular}{l|l}
\hline \multicolumn{1}{c|}{ Selected Task } & Tasks selected \\
\hline Factor 1 & Internet searching \\
\hline Factor 2 & Subway map \\
\hline Factor 3 & Memo \\
\hline Factor 4 & Gallery (photo, video) \\
\hline Factor 5 & Application store \\
\hline
\end{tabular}

\subsection{Tasks}

The first experiment involved the use of a uni-mode for five single tasks containing few sub-tasks in Table 3 . Tasks 1 and 3 consist of text entry which helps to verify the effects of each input mode characteristics. Task 2 is pointing task on a narrow area and navigation in spatial space. Compared with other tasks, Task 4 is differentiated by the simplicity of the task. Task 5 is downloading applications and selecting a list menu selection. Tasks used in the experimentare shown in Figure 1.

Table 3. Results of Sub tasks

\begin{tabular}{l|l}
\hline \multicolumn{1}{c|}{ Task } & \multicolumn{1}{c}{ Sub task } \\
\hline Task 1 (Internet searching) & Text entry $->$ Selection \\
\hline Task 2 (Subway map) & $\begin{array}{l}\text { Naviaation 1 (Spatial info) -> Pointing 1 -> Navigation 2 (Spatial info)-> Pointing 2 } \\
->\text { Selection }\end{array}$ \\
\hline Task 3 (Memo) & Selection $->$ Text entry $->$ Selection \\
\hline Task 4 (Gallery) & Navigation 1 (Spatial info) -> Selection \\
\hline Task 5 (Application store) & Text entry $->$ Selection $->$ Selection (List menu) -> Selection \\
\hline
\end{tabular}

\section{Uni-Mode Experiment}

The purpose of the first experiment was to verify uni-mode effects by smart phone function characteristics through usability test. Uni-mode experiment is using only one mode when performing tasks. This experiment was conducted to identify the level of preference of modes according to the characteristics of the task. 


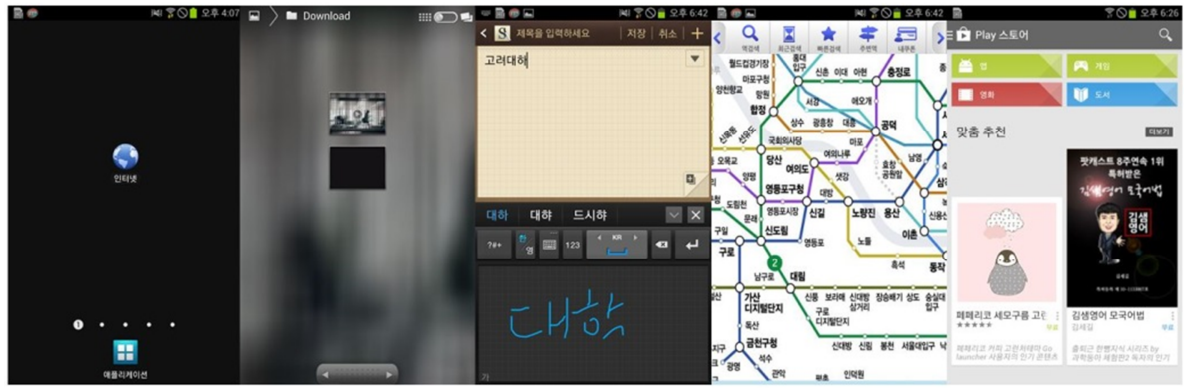

Figure 1. Tasks 1 through 5 used in the experiment in the respective order

\subsection{Subject}

A total of fourteen participants volunteered in the experiment. The participants consisted of males and females whose ages ranged from 20 to 30 years old (Average $=29.7$ and SD $=4.31$ ). All of them have used smart phones for three years or longer. All had normal vision and no dysfunction using their arms and hands.

\subsection{Experiment apparatus and setting}

In order to evaluate full-factorial experimental design, voice mode was designed using the Mobizen Software that provided a technical remote support to mobile devices on the monitor. The resolution of screen was $1,290 \times 800$ pixels.

\subsection{Experimental design}

The dependent variables were user preference and task completion time. The independent variable in the first experiment was the type of modes (i.e., touch, pen, or voice), while the independent variable in the second experiment was the type of tasks (i.e., Internet searching, subway map, memo, gallery [photo, video], and app market). User preference was measured using a 9-point

Table 4. Variables of uni-mode experiment

\begin{tabular}{|c|c|c|}
\hline \multicolumn{2}{|c|}{ Variables } & Description \\
\hline \multirow{8}{*}{ Independent variables } & \multirow{3}{*}{ Mode type } & Touch \\
\hline & & Pen \\
\hline & & Voice \\
\hline & \multirow{5}{*}{ Task type } & Internet searching \\
\hline & & Subway map \\
\hline & & Memo \\
\hline & & Gallery (photo, video) \\
\hline & & Application store \\
\hline \multirow{2}{*}{ Dependent variable } & Objective measurement & Task completion time \\
\hline & Subjective measurement & Subjectivepreference \\
\hline
\end{tabular}


scale and selected variables are shown in Table 4.

\subsection{Procedure}

The experimental procedure consisted of four phases: preparation, practice, main experiment, and subjective rating. In the preparation phase, the experimenter collected each participant's information, explained the purpose, procedure and task of the experiment, and gave general instruction. Multi-screen wi-fi connection was confirmed for the environment. In the practice phase, the participants were given sufficient time to practice mode transition because most participants were not familiar with voice and pen mode type. In main experiment phase, the participant performed the given task. During the execution of experimental tasks, the smart phones were placed where the each participants felt comfortable to manipulate the phones.

\subsection{Results of the Uni-Mode Experiment}

\subsubsection{Task completion time}

The Analysis of variance (ANOVA) was conducted to find significant main effects and interactions between task and mode types The result of ANOVA is illustrated in Table 5. Mode $(p<.001)$ and task $(p<.001)$ were both statistically significant. There was also an interaction between mode and task in Figure 2. It means task completion time was affected by mode. Figure 3 shows the average of task completion times. As for task completion time, voice appeared to require less time in most of tasks. However, with the exception of Tasks 2 and 4, task completion time of voice was higher than the other mode. The reason for this would be that Tasks 2 and 4 are based on Name-tag. For the task based on text-entry (Internet, Memo, App market), task completion time for

Table 5. ANOVA result of task completion time

\begin{tabular}{l|c|c|c|c|c}
\hline & DF & SS & MS & $F$-value & $P$-value \\
\hline Mode & 2 & 1422.20 & 711.10 & 93.03 & $0.000^{\star}$ \\
\hline Task & 4 & 5161.55 & 1290.38 & 148.39 & $0.000^{\star}$ \\
\hline Mode*Task & 8 & 1184.27 & 148.03 & 20.72 & $0.000^{\star}$ \\
\hline
\end{tabular}

*: Significant at $\alpha=0.05$

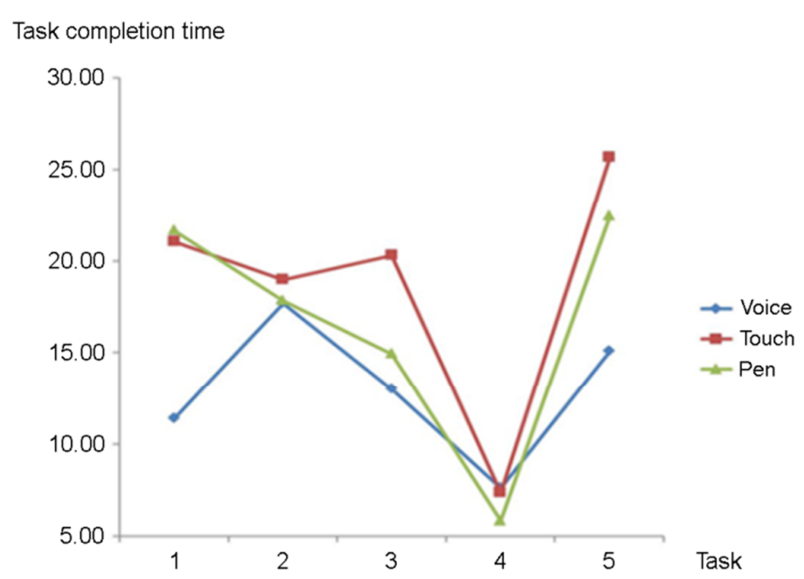

Figure 2. Interaction between mode and task 
voice was shorter than others.

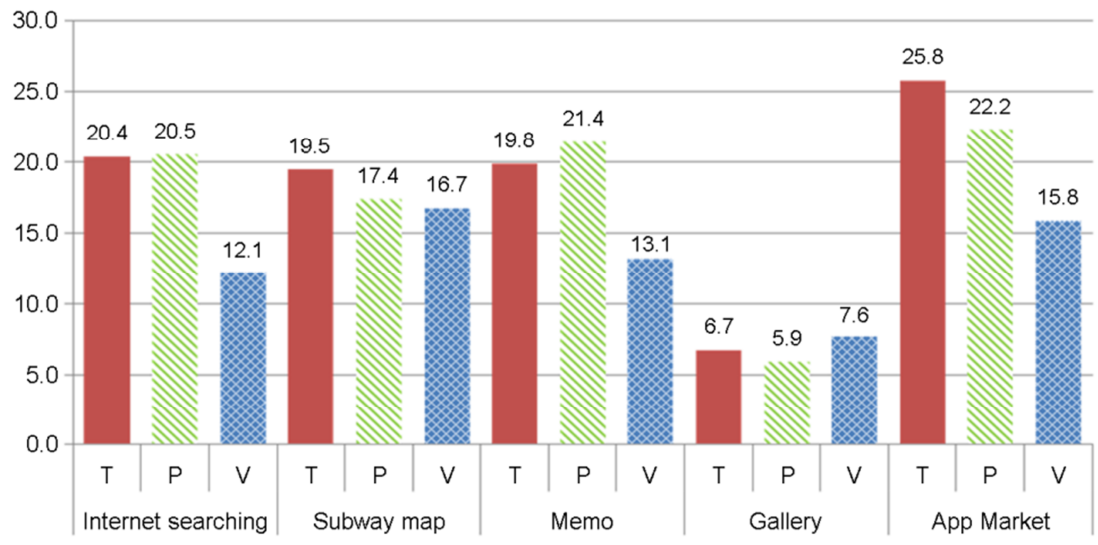

Figure 3. Task completion times by task and mode

\subsubsection{Subjective preference}

Task and the interaction between task and mode were both statistically significant $(p<.001)$ but mode was not statistically significant. The result of ANOVA is illustrated in Table 6.

Table 6. ANOVA result of subjective preference

\begin{tabular}{l|c|c|c|c|c}
\hline & DF & SS & MS & F-value & $P$-value \\
\hline Mode & 2 & 4.067 & 2.033 & 1.281 & 0.295 \\
\hline Task & 4 & 7.305 & 1.826 & 4.234 & $0.005^{\star}$ \\
\hline Mode*Task & 8 & 17.410 & 2.176 & 5.765 & $0.000^{\star}$ \\
\hline
\end{tabular}

*: Significant at $\alpha=0.05$

In tasks 1 and 2, voice was highly preferred. There was nostatistical difference in mode preference in Tasks 2 and 4 . Each Task showed different order of preference, as seen in Figure 5, showingthat there is an interaction between mode and task. Even though touch and pen have different properties, there was no significant differences in preference. However, the preference for pen was much higher in Task 3 as shown in Figure 4. Even though touch and pen are in the same motion-based categories, mode preference could change according to the task. Touch has the lowest score in Task 2, but it was most preferred in Task 5. Compared to the other mode type, pen's preferences showed a larger difference between the scores with different tasks. Even though Tasks 1 and 5 hadthe same text entry sub-tasks, the result of preferred modes were different. Due to interaction, preferred modes in each task were again derived from expert opinion and additional frequency analysis. Through the frequency analysis, it was found that there was no difference in mode characteristics. Table 7 is the results of preferences in mode type. 


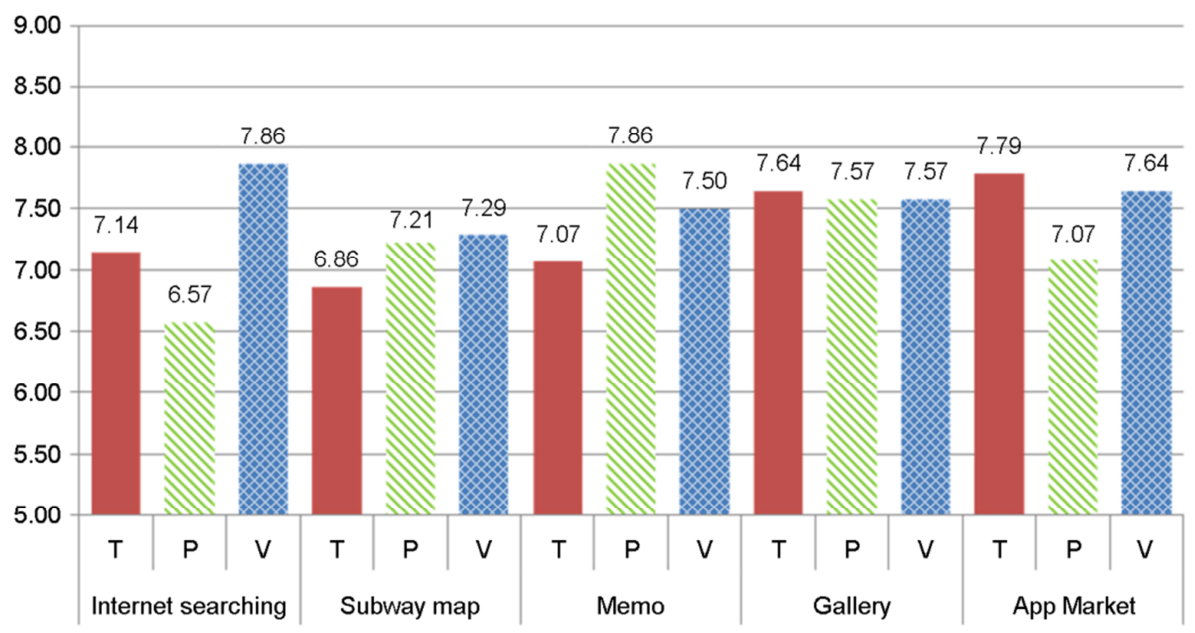

Figure 4. Subjective preference of tasks by mode

Subjective preference

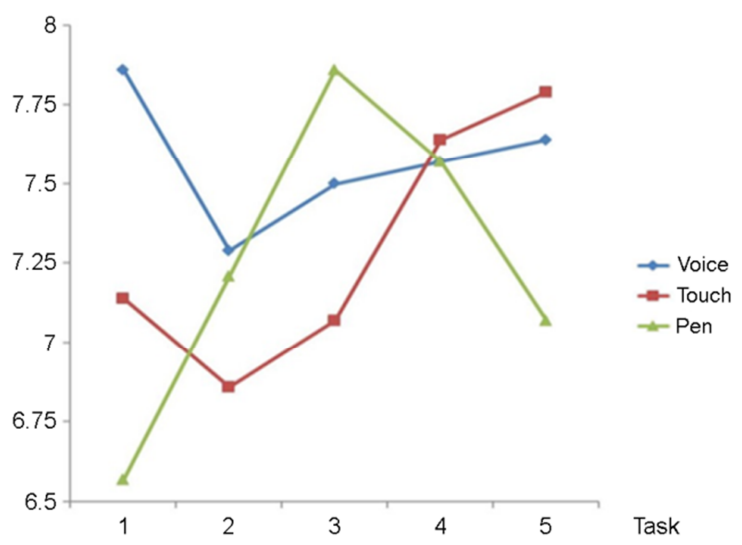

Figure 5. Interaction between mode and task

Table 7. Results of mode preference

\begin{tabular}{l|c|c}
\hline \multirow{2}{*}{\multicolumn{1}{c|}{ Task }} & \multicolumn{2}{c}{ Preferred mode type } \\
\cline { 2 - 3 } & Most preferred & Second preferred \\
\hline Task 1 (Internet searching) & Voice & Touch \\
\hline Task 2 (Subway map) & Voice & Ven \\
\hline Task 3 (Memo) & Pen & No difference \\
\hline Task 4 (Gallery) & \multicolumn{2}{c}{ Voice } \\
\hline Task 5 (App Market) & Touch & \multicolumn{2}{c}{} \\
\hline
\end{tabular}




\section{Multi-Mode Experiment}

The second experiment examined the use of a multi-mode for three dual tasks. The dependent variables were again user preference and task completion time. The independent variable in the second experiment was the type of tasks (i.e., internet searching, subway map, memo, gallery, and application store).

\subsection{Experimental design}

In multi-mode experiment, new tasks were made by combining the results of uni-mode experiment. Task 1 was excluded since it was the same as Task 5's sub-task (Text-entry). Task 4 was also excluded since there was no difference observed between modes. Three new tasks (Tasks 2, 3, 5) were made and used for multi-mode experiment.

Subjects who participated in this experiment were the same participants from uni-mode experiment under the identical experimental conditions. The dependent variables were user preference and task completion time. The independent variable in the multi-mode experiment was the type of task as shown in Table 8.

Table 8. Combined tasks of experiment

\begin{tabular}{|c|c|c|c|c|c|c|}
\hline \multirow{2}{*}{$\begin{array}{l}\text { Combined tasks } \\
\text { Mode sequence } 1\end{array}$} & \multicolumn{2}{|c|}{ Subway map + App market } & \multicolumn{2}{|c|}{ App market + Memo } & \multicolumn{2}{|c|}{ Memo + Subway map } \\
\hline & Touch & Touch & Touch & Touch & Touch & Touch \\
\hline Mode sequence 2 & Touch & Voice & Touch & Voice & Touch & Voice \\
\hline Mode sequence 3 & Touch & Pen & Touch & Pen & Touch & Pen \\
\hline Mode sequence 4 & Pen & Touch & Pen & Touch & Pen & Touch \\
\hline Mode sequence 5 & Pen & Voice & Pen & Voice & Pen & Voice \\
\hline Mode sequence 6 & Pen & Pen & Pen & Pen & Pen & Pen \\
\hline Mode sequence 7 & Voice & Touch & Voice & Touch & Voice & Touch \\
\hline Mode sequence 8 & Voice & Voice & Voice & Voice & Voice & Voice \\
\hline Mode sequence 9 & Voice & Pen & Voice & Pen & Voice & Pen \\
\hline
\end{tabular}

\subsection{Results of the Multi-mode experiment}

\subsubsection{Task completion time}

The result of ANOVA is illustrated in Table 9. Mode and combined tasks were analyzed to be statistically significant at $p<.001$. There was also an interaction between mode and task. Figure 6 shows the distribution of average task completion times using the combination of two different input modes. In terms of completion time, combined tasks 1 and 2, the combination of touch and pen consumed more time than other combination of input modes.

\subsubsection{Subjective preference}

The ANOVA result for subjective preference of task and mode types in Table 10 showed that main effects were significant $(p<.005)$. 
In combined task 1 , voice-voice $(\mathrm{V})$ resulted in faster completion time and most preferred. Figure 7 shows that voice uni-mode (VV) and pen-voice (PV) were preferred in Task 2. In task 3, touch-voice (TV) and pen-voice (PV) were preferred.

Table 9. ANOVA result of the task completion time

\begin{tabular}{l|c|c|c|c|c}
\hline & DF & SS & MS & $F$-value & $P$-value \\
\hline Mode & 8 & 7115.174 & 888.464 & 137.582 & $0.000^{\star}$ \\
\hline Task & 2 & 235.444 & 117.722 & 13.957 & $0.000^{\star}$ \\
\hline Mode*Task & 16 & 1688.032 & 105.502 & 15.170 & $0.000^{\star}$ \\
\hline
\end{tabular}

*. Significant at $\alpha=0.05$

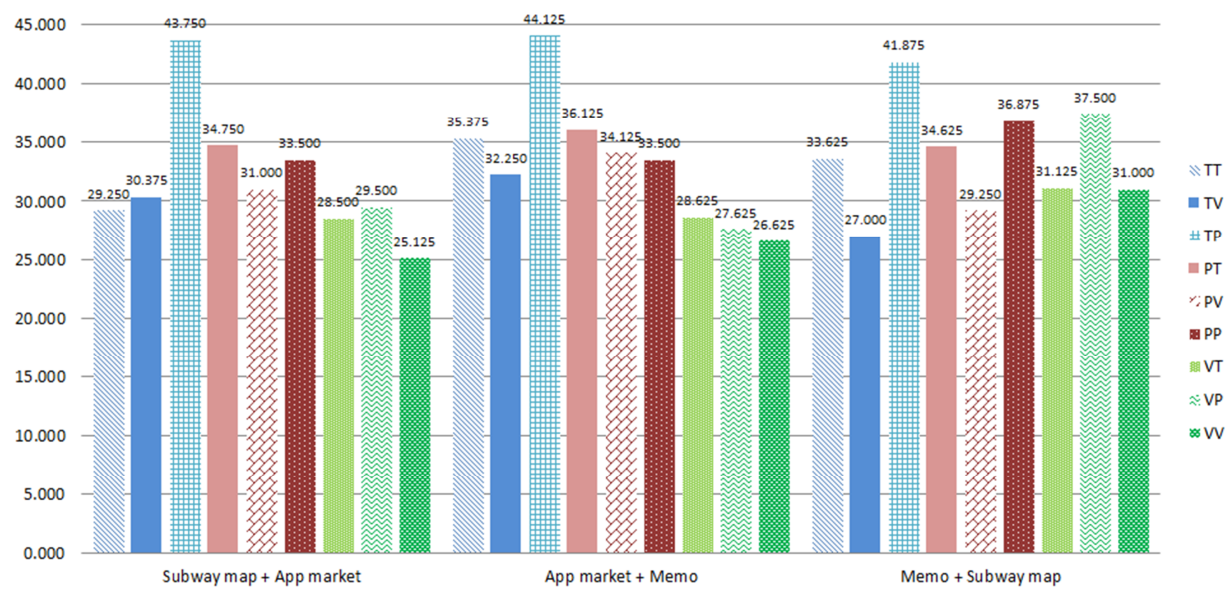

Figure 6. Task completion times by task and mode

Table 10. ANOVA result of the subjective preference

\begin{tabular}{l|c|c|c|c|c}
\hline & DF & SS & MS & $F$-value & $P$-value \\
\hline Mode & 8 & 318.952 & 39.869 & 34.108 & $0.000^{*}$ \\
\hline Task & 2 & 4.778 & 2.389 & 3.383 & $0.035^{\star}$ \\
\hline Mode*Task & 16 & 61.460 & 3.841 & 3.964 & $0.000^{*}$ \\
\hline \multicolumn{7}{r|}{}
\end{tabular}

The multiple input modes of the vocal-motion pointer combination were better within the one-time mode change, regardless of the sequence of the mode change. In all tasks, preference has been underestimated if entering the voice mode first and changing to gesture-based mode. The average user preference is shown in Figure 7. All preferred modes had a common fact that they contained voice in the second input mode. Student-Newman-Keuls post analysis for each taskwas conducted in Table 11. The preferred mode for the combined task was shown in Table 12. 


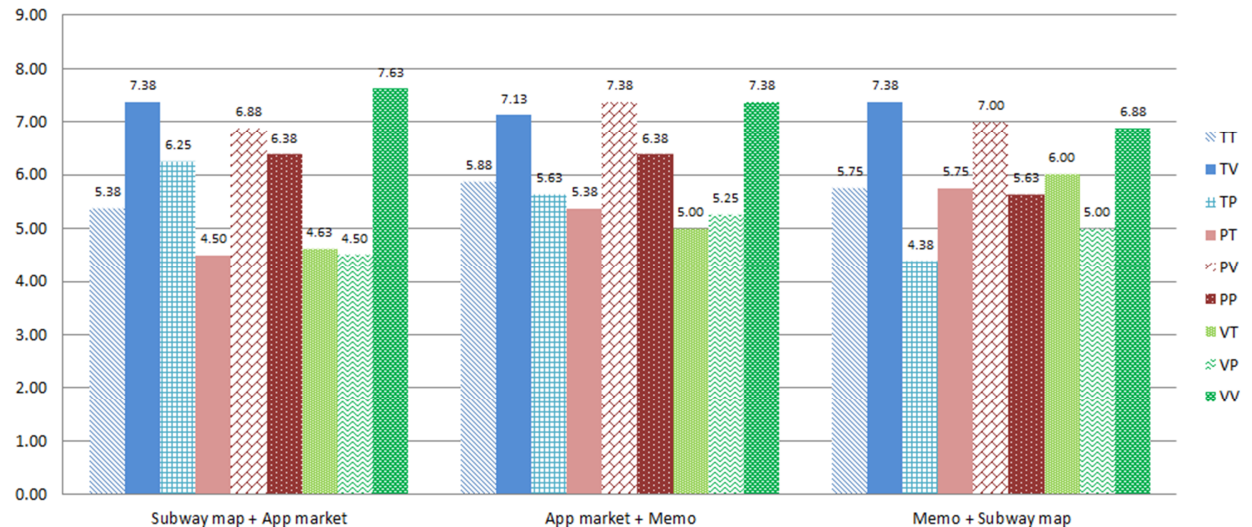

Figure 7. Subjective preference of tasks

Table 11. The SNK results of Multi-mode tasks

\begin{tabular}{l|l|c|c|c}
\hline \multicolumn{2}{c|}{ Mode } & New task 1 & New task 2 & New task 3 \\
\hline Touch & Touch & B & B \\
\hline Touch & Voice & A & A & A \\
\hline Touch & Pen & B & C & B \\
\hline Pen & Touch & C & A & B \\
\hline Pen & Voice & A & B & B \\
\hline Pen & Pen & B & A & A \\
\hline Voice & Touch & C & C & C \\
\hline Voice & Voice & A & C & C \\
\hline
\end{tabular}

Table 12. Preferred mode type of combined tasks

\begin{tabular}{|c|c|c|c|}
\hline & New task & & \\
\hline \multirow{3}{*}{1} & \multirow{3}{*}{ Subway map + App Market } & Voice & Voice \\
\hline & & Touch & Voice \\
\hline & & Pen & Voice \\
\hline \multirow{3}{*}{2} & \multirow{3}{*}{ App Market + Memo } & Voice & Voice \\
\hline & & Pen & Voice \\
\hline & & Touch & Voice \\
\hline \multirow{3}{*}{3} & \multirow{3}{*}{ Memo + Subway map } & Touch & Voice \\
\hline & & Voice & Voice \\
\hline & & Pen & Voice \\
\hline
\end{tabular}




\section{Discussion}

In multi-mode experiment, the preferred combination of mode and the order of the alternatives are selected through uni-mode experiments. In addition, the ANOVA result of the multi-mode experiment showed significant main effects of preference and completion time. When a variety of input-modes was introduced into a smartphone with the development of technology, Inputmodes were independently designed without considering multi-tasking. There may be a limit to verify all existing input-modes (Touch, pen, voice, gesture, eyeMovement, facial expression, fingerprint, etc.) since this study selected only three (Touch, pen, voice). However, this study showedthat the order of mode combination may affect the usability of multi-modes. Therefore, when designing a multi-modal system, the fact that there are frequent transitions between various mobile contents in different modes should be properly considered.

\subsection{Mode switching in multi-mode}

Reeves et al. (2004) stated that the designer should support the best modality or combination of modalities in changing mobile environment. In changing environment, the combination of pen and voice permits flexible use of input modes, including alternation and integrated use. In this study, the order of mode combinations affected the usability of multi-modes.

Since in reality, input-modes in current smart phones are independently designed, designers are not able to consider the transition in between modes. Although some of contents in smartphones include switching mode, they focused not on main mode switching but switching in sub-tasks.

In fact, a non-preferred combination of uni-mode could be a good alternative when it is combined with another and provided as multi-mode. This means that providing a variety of input-mode helps the user understand how to interact with the system.

In this study, the effect of multi-modes was examined only in sequential processing. Further study is necessary for verifying the effects and the order of mode switching. The combination of input modes has influence on the preference and consistency of user performance. It means that there was a preferred mode switching order such as a combination of touch and voice or that of pen and voice. Stifelman et al. (1993) stated that providingthe combination of two complementary input modalities allows the user to task advantage of the different capabilities provided by each input modality since it allows the limitation of one type of input to be overcome by the other.

\subsection{Combination of input modes}

With a limitation of present voice interface, the scope of the work presented in this paper is limited by the interaction design of the experimental prototypes. Furthermore, the present voice interface has a technical limitation in switching mode especially in an interface menu structure. The Yankelovich (1995) also recommended that the design of voice interface should consider the combination of mode types. Combination of voice type shows high preference among the users. However, the order of voice is significantly important in usability. The result of this study shows that voice mode is very versatile and can be successfully combined with any other input modes.

When it comes to completion time, combination of pen and touch takes long time while switching a mode. There was no difference between the use of pen and touch since they were both motion-based type modes. Therefore, same mode types resulted in low preference in switching mode. 


\section{Conclusion}

There were significant results based on the user's preference in mode switching. This study considered multi-modal interactions from a user's point of view when using a wide range of input-modality and content from the smart phone environment. It may be utilized as an user-centered design guideline for mobile multi-modal UI system.

\section{References}

Oviatt, S. and Olsen. E., Integration themes in multimodal human-computer interaction, Proceedings of the 3rd International Conference on Spoken Language Processing (ICSLP). Yokohama, Japan. 1994.

Oviatt, S., Ten myths of multimodal interaction, Communications of the ACM, 41(11), 74-81, 1999.

Oviatt, S., Cohen, P., Wu, L., Duncan, L., Suhm, B., Bers, J., Holzman, T., Winograd, T., Landay, J., Larson, J. and Ferro, D., Designing the user interface for multimodal speech and pen-based gesture applications: state-of-the-art systems and future research directions. Human-computer interaction 15(4), 263-322, 2000. doi:10.1207/S15327051HCI1504_1

Park, S.S., Park, S.C., Ahn, S.Y., Kim. W.W. and Koo, M.W., Design and Implementation of Multimodal Middleware for Mobile Environments, Korean Society of Speech Sciences, 60, 125-144, 2006.

Reeves, L.M., Lai, J., Larson, J.A., Oviatt, S., Balaji, T.S., Buisine, S., Collings, P., Cohen, P., Kraal, B., Martin, J.C., McTear, M., Raman, T., Stanney, K.M., Su. H. and Wang, Q.Y., Guidelines for multimodal user interface design. Communications of the ACM, 47(1), 5759, 2004.

Stifelman, L.J., Arons, B., Schmandt, C. andHulteen, E.A., Voice Notes: a speech interface for a hand-held voice note taker. Proceedings of the INTERACT'93 and CHI'93 conference on Human factors in computing systems. 179-186, New York, NY, 1993.

Trouvain, B. and Christopher, M., A comparative study of multimodal displays for multi robot supervisory control. Engineering Psychology and Cognitive Ergonomics, Volume 4562, 184-193, 2007.

Wickens, C.D., Multiple resources and performance prediction. Theoretical issues in ergonomics science 3(2), 159-177, 2002. doi:10.1080/14639220210123806

Yang, J.Y. and Jung, E.C., Model Development for multi-tasking and multi-modality through a case analysis of mobile devices, Society of Korea Design Trend, 37, 277-288, 2012.

Yankelovich, Nicole, Gina-Anne Levow, and Matt Marx., Designing Speech Acts : Issues in Speech User Interfaces.Proceedings of the SIGCHI conference on Human factors in computing systems, 369-376, New York, NY, 1995.

Yu, J., Kim, S., Choe, J. and Jung, E.S. Multi-Modal Controller Usability for Smart TV Contro/Journal of the Ergonomics Society of Korea, 32(6), 517-528, 2013. 


\section{Author listings}

Gaeun Lee: leegganni@gmail.com

Highest degree: BA, Department of Industrial design \& Information, Korea University

Position title: MS, Candidate, Department of Industrial Management Engineering, Korea University

Areas ofinterest: Product design, UX, UI

Seongmin Kim: iampreppie@korea.ac.kr

Highest degree: MS, Candidate, Department of Industrial Management Engineering, Korea University

Position title: PhD, Candidate, Department of Industrial Management Engineering, Korea University

Areas of interest: Product Development

Jaeho Choe: jhchoe@daejin.ac.kr

Highest degree: PhD, Department of Industrial Engineering, POSTECH

Position title: Professor, Department of Industrial \& Management Engineering, Daejin University

Areas of interest: Product design, UX, Affective engineering

Eui Seung Jung: ejung@korea.ac.kr

Highest degree: $\mathrm{PhD}$, Department of Industrial Engineering, Pennsylvania State University

Position title: Professor, Department of Industrial Management Engineering, Korea University

Areas ofinterest: Product Development, Technology Management 